\title{
Protée
}

\section{Pour une théorie générale des figures}

\section{Michel Meyer}

Volume 38, numéro 1, printemps 2010

Le Groupe $\mu$ entre rhétorique et sémiotique

URI : https://id.erudit.org/iderudit/039699ar

DOI : https://doi.org/10.7202/039699ar

Aller au sommaire du numéro

Éditeur(s)

Département des arts et lettres - Université du Québec à Chicoutimi

ISSN

0300-3523 (imprimé)

1708-2307 (numérique)

Découvrir la revue

Citer cet article

Meyer, M. (2010). Pour une théorie générale des figures. Protée, 38(1), 19-25. https://doi.org/10.7202/039699ar

\section{Résumé de l'article}

La théorie des figures a-t-elle une rationalité sous-jacente ? Où la trouver ? La problématologie ou théorie du questionnement est la clé de ce problème. Sa dette à l'égard du Groupe $\mu$ tient à la mise en évidence de quatre opérations fondamentales : le =, le \pm , le +, et le -, ou répétition-substitution, modification, ajout et négation. On les retrouve dans les figures de langage et les figures de pensée, celles-ci devant traduire une problématicité qui va de la plus faible, avec les figures de son, à la plus forte, avec les figures de pensée, où le problème est dit mais comme résolu. 


\section{POUR UNE THÉORIE GÉNÉRALE DES FIGURES}

MICHEL MEYER

\section{1. À QUOI SERVENT LES FIGURES?}

Nous pensons rarement sans utiliser des figures de style, et nous ne nous exprimons jamais sans y recourir. Le sens littéral de nos propos semble consister en des figures qui se sont usées et restreintes au fil du temps, ne laissant plus apparaître qu'un seul sens, celui qui est le plus communément admis, expurgeant par là l'image au profit du concept et de l'idée. Parler du "pied de la lettre» n'évoque pas plus un pied que le «crépuscule de la vie» n'éveille l'image du soleil qui se couche. Ce genre de phrases fait exactement penser à ce à quoi elles font penser, comme si les figures avaient acquis, par l'usage, une littéralité qu'elles n'avaient pas au départ. La littéralité serait même ce processus de restriction et d'épuration à une seule signification, qui ferait oublier l'étymologie imagée des mots qu'on emploie dans la vie de tous les jours. Qu'est-ce qui est alors premier, les sens pluriels, qui s'appauvrissent avec le temps ou qu'on sélectionne aisément au vu du contexte, ou le sens littéral, dont on hériterait d'emblée avec la maîtrise d'une langue? Les métaphores seraient pour plus tard, les jeux de mots aussi, comme tous les effets de style d'ailleurs.

Peut-être que ce problème est insoluble, parce qu'il est mal posé. La figurativité n'est ni première ni seconde par rapport au sens univoque et clair qui, seul, permet de comprendre ce que les autres nous disent. Leur interaction n'est pas de cet ordre déterminé, ce qui est premier et ce qui est dérivé n'a pas grand intérêt pour résoudre cette question. Il faut reprendre le problème sur d'autres bases. Deux grandes visions existent aujourd'hui à propos des figures, qu'il va falloir compléter, modifier, amender, pour les intégrer dans une perspective plus englobante, celle de la rhétorique générale du Groupe $\mu$ et l'approche de G. Molinié, qui oppose les figures macrostructurales et les figures microstructurales, fondées sur l'aspect linguistique du mot ou de la phrase. Certes, on a traité des figures de rhétorique bien avant, dès l'Antiquité grecque et romaine. Mais cela a souvent donné lieu à des catalogues un peu arbitraires, où les classifications s'opposent aux classifications, sans qu'on puisse trancher en faveur d'une ligne directrice qui s'imposerait de toute évidence. Il reste alors bien des questions en suspens. Combien y a-t-il de tropes, et quels sont ceux qui sont les plus fondamentaux? Et que choisir comme figures de sons ou de mots, comme figures grammaticales ou de construction, comme tropes ou comme figures de pensée? Et d'ailleurs, pourquoi s'en tenir à cette classification en quatre groupes? D'où provient-elle? Trente, quarante 
figures peuvent émailler le catalogue, et Dumarsais, en 1730, ou Fontanier, en 1830, tombaient encore en désaccord sur le nombre de figures et les classifications pertinentes. Si l'on remonte à Aristote, celui-ci voyait dans la métaphore l'essence même de la figurativité, le Groupe $\mu$, au XXe siècle, lui préférait la synecdoque. À l'âge des Lumières, G. Vico avait choisi de s'arrêter à quatre tropes exemplaires, la métaphore, la métonymie, la synecdoque et l'ironie, où il voyait les quatre moments clés de l'Histoire se cristalliser. Il y a d'abord l'âge des dieux, qui identifie les forces et les êtres sur base d'indices communs soi-disant essentiels, prescrivant aux êtres, aux choses, et aux dieux, une identité qui n'était guère évidente ni apparente, ce qui est le propre de l'identité métaphorique, comme la force et le feu, ou le faucon et le pharaon. Vient ensuite l'âge des héros qui, obnubilé par la grandeur aristocratique de certains, choisit la métonymie pour exprimer, de façon exemplaire, la propriété sensée résumer toutes les autres, comme lorsqu'on dit aujourd'hui que Hugo est une grande plume, pour dire que c'est un grand écrivain. La plume est symbolique de l'écriture. Vient ensuite l'âge des hommes, où l'égalité veut que ce soient les généralisations qui importent. Elles font penser à une sorte de démocratisation qui se généralise au conceptuel dans le langage et la pensée. C'est le règne de la synecdoque, qui identifie le particulier par le général; c'est l'âge de la science. Et l'on a enfin l'âge de la prise de conscience de tout ce processus historique qui vient d'être évoqué, par lequel on se met à distance du rhétorique, pour le démystifier, comme prétend y parvenir Vico. La rhétorique comme conception du monde doit ainsi dépasser l'âge de la rhétorique. Il est vrai que l'accélération de l'Histoire délittéralise les vérités d'hier, qui cessent d'être valables, et leur rhétorisation permet d'en prendre conscience. Elles ne sont plus alors que des métaphores de réponses, auxquelles certains s'accrochent encore, tandis que d'autres savent qu'elles sont devenues problématiques, comme toute métaphore qui finit par devenir énigmatique avec l'usure de l'Histoire. Il faut alors de nouvelles réponses pour les remplacer. D'où le conflit des Anciens et des Modernes, qui traverse régulièrement l'histoire des hommes.
Les figures servent donc à marquer la différence du littéral et du figuré, qu'il faut entendre elle-même comme une figure de la différence question-réponse, appelée aussi différence problématologique.

Le Groupe $\mu$ a mis le doigt sur un fait aussi incontournable que capital en rhétorique. Celle-ci s'appuie toujours sur quatre opérations, qui vont de l'identité à l'opposition, en passant par diverses modalités de différenciation, comme l'adjonction d'une réponse nouvelle ou la modification de celle apportée. Ces opérations de base font subir des variations à ce qui est proposé, selon une loi de différenciation croissante, qui va de la répétition (approbatrice) à la différence oppositionnelle (de désaccord), si ce n'est que l'identité et la différence en rhétorique ne signifient pas forcément l'accord ou le désaccord, sauf à faire le lien avec des lieux d'approbation et de désapprobation préalables, dont la réponse nouvelle se démarquerait ou non. Il faut donc nuancer le propos, et bien scinder le composant rhétorique formel, de son impact argumentatif, qui relève de la réaction de l'auditoire: une identité peut choquer (si elle s'accorde avec une valeur honnie) et une différence peut plaire (si elle confirme ce que l'on aime). À cela s'ajoute une autre caractéristique. L'identité et la différence, dans leur aspect formel d'écart et de variation, se retrouvent en fait dans les figures de son, de grammaire, de pensée, ou dans les tropes. L'auditoire et sa réaction ne jouent aucun rôle là-dedans. La métaphore traduit l'identité, l'ironie, l'opposition ou la forte différence, la synecdoque ajoute, la métonymie modifie. La métaphore traduit une certaine identité, car son but est d'identifier les choses et les êtres au nom d'une propriété singulière, comme l'ironie vise à souligner l'opposition, malgré une identité qui n'est plus que de pure forme (comme lorsqu'on affirme «c'est malin!» pour signifier le contraire). On peut évidemment se demander pourquoi l'on a ces quatre opérations de base, et non pas cinq ou huit, et celles-là et non pas d'autres. Làdessus, le Groupe $\mu$ semble muet. Car, pour donner l'explication qui aurait convenu, il eût fallu défendre une rhétorique problématologique, où ces opérations de base représentent les modalités de réponse, donc d'affirmation sur ce qui est pertinent pour traiter 
une question rhétoriquement. Cela exige qu'on aille au-delà d'une simple théorie de la figurativité. Et là, on n'est plus dans la technique rhétorique, mais dans la théorisation du champ lui-même. D'où la question qu'il faut se poser: comment concevoir la rhétorique? La rhétorique est la négociation de la distance entre les individus sur une question donnée. Cette question traduit ce qui sépare orateurs (éthos) et auditoires (pathos), et cela peut également les réunir, si la question est conventionnelle, comme lorsqu'on parle du temps qu'il fait ou des enfants. Mais dans tous ces cas, néanmoins, ce dont on discute est une question, qui est plus ou moins problématique, et donc parfois qui ne l'est pas du tout, pour ne pas vexer, comme on le voit dans les formules de politesse. Ce dont on discute est ce dont il est question. Le discours de politesse, qui est un logos peu conflictuel, crée du liant social entre les êtres qui se connaissent peu ou pas du tout, et les questions se veulent alors consensuelles. À l'inverse, il y a des questions à haute densité problématique, comme celles de la politique, qui sont souvent source de conflits et de débats. Dans le discours épidictique, où le problème est de faire en sorte qu'il n'y ait pas de problème, le discours est des plus convenus. Dans l'oraison funèbre, par exemple, le défunt est toujours présenté comme plein de qualités, réelles ou supposées, ce dont il est question ne devant pas faire problème en une circonstance aussi grave que la mort.

Le figuré n'est finalement pas plus premier que le littéral, et inversement. L'articulation des deux se fait simultanément, pour indiquer un renvoi, une suggestion, voire une inférence: une réponse appelle une autre réponse. Dans le trope, la réponse, par sa seule forme, demande elle-même d'effectuer ce dépassement. C'est ce problème-là qu'elle communique à autrui, par une sorte de dédoublement dans le répondre même. Vouloir dire autre chose par ce que l'on dit, c'est mettre en question la littéralité ou, plutôt, la concevoir comme une question adressée à autrui pour la circonstance, et comme dans le trope, cela s'effectue par la forme, où la lecture littérale est explicitement impossible. Un propos qui n'est que littéral n'est autre qu'une réponse qu'on considère comme affirmant exactement ce qu'elle dit en tant que réponse. C'est un point d'arrêt. Si l'on affirme
"Il est une heure», cela peut vouloir dire exactement cela, et rien de plus, à savoir l'heure qu'il est, parce qu'on l'a demandé tout simplement. Il n'y a donc rien à ajouter. L'échange prend fin. Mais si à la fin d'un exposé, je dis «Il est une heure», alors que personne ne m'a rien demandé, c'est que j'ai voulu exprimer autre chose qu'un simple constat horaire. C'est une autre question que l'heure qu'il est qui se trouve suggérée, et cela donne lieu à une interrogation, souvent rapidement effectuée, qui a trait à la question réelle qui est sous-jacente dans celle que j'ai abordée. «Pourquoi parle-t-il de l'heure?» vont se demander les autres. Je cherche en fait à leur signifier autre chose, qu'ils sont invités à inférer. Ils vont devoir se figurer ce que j'ai voulu dire, en interrogeant ma réponse. Ils se rendent ainsi parfaitement compte que celle-ci doit donc répondre à une autre question que celle littérale de l'heure, que personne n'a soulevée. En l'occurrence, vu les usages en vigueur, il s'agit de savoir si ce n'est pas le moment de passer à table, ou simplement d'arrêter l'exposé. Mon dire pose question, et c'est à mon interlocuteur de répondre, "C'est cela, passons à table", par exemple. D'où la loi fondamentale de la rhétorique $\mathrm{r}_{1} \rightarrow \mathrm{q}_{1} \cdot \mathrm{q}_{2}$. Une réponse présente un sens figuré, si elle répond littéralement à une autre question, laquelle lui donne son vrai sens, d'où le renvoi à $\mathrm{q}_{2}$.

Les figures servent à soulever, à aborder des questions, de façon indirecte, implicite. Le langage figuratif est au service de cette tâche indispensable, qui consiste à communiquer avec un ensemble de réponses finies une multitude d'autres réponses qui s'imposent, aux yeux de l'orateur en tout cas, dans certains contextes où il est préférable de ne pas tout préciser, où cela n'est même guère utile. Par délicatesse pour autrui et ses facultés, par économie, pour éviter des contradictions à n'en plus finir, par subtilité, ou tout simplement parce qu'on ne peut ni ne veut tout dire, comme en littérature, où le sens doit pouvoir survivre à l'intention de l'auteur.

Mais ces opérations élémentaires,,,$+-=$, et \pm , qui définissent les réponses rhétoriques possibles à une proposition qui fait question pour l'auditoire, ne définissent pas la nature des figures, leurs différences, ni leur nombre, car on retrouve ces opérations à tous 
les niveaux de la figurativité. D'où la nécessité de faire intervenir un autre principe.

\section{COMBIEN Y A-T-IL DE CLASSES DE FIGURES ET POURQUOI?}

À côté des figures de mots, qui jouent sur les sons, on trouve les figures de mots qui s'attachent au sens par le biais de la grammaire: l'inversion du sujet et du verbe ou du complément, les répétitions ou les ellipses, par exemple, sont autant de moyens pour mieux souligner ce dont il est question dans une réponse qui ne le (ou la) dit pas explicitement. La rhétorique présente les questions qui divisent, ou qui unissent, et elle le fait par le biais de réponses, et non en partant explicitement des questions, comme en droit, qui est un processus argumentatif et conflictuel. Cette démarche, qui consiste à offrir des réponses à des questions qui semblent ainsi ne plus ou ne pas se poser, a un effet rhétorique immédiat, et voulu: faire comme si elles étaient abolies, qu'elles avaient disparu par enchantement, sous l'effet de la magie du style ou de l'élégance formelle du propos. La beauté du discours, les effets de style, la forme en somme, permettent de donner cette impression. Les questions en question ne se posant plus, elles semblent résolues de ce fait même. Évidemment, il est d'autant plus facile d'y parvenir que les questions présentent une faible densité problématique. Quand un problème réel et aigu se pose, le jeu de mots ou la manipulation formelle de la phrase ne sauraient suffire. C'est pour cela que l'esprit humain a mis en œuvre un troisième genre de figures, les tropes. Le propre des tropes, comme les métaphores par exemple, est de présenter une énigme de par la forme littérale du discours. Le répondre énonce indirectement une question, qui n'est résolue cette fois que rhétoriquement. "Richard est un lion", alors qu'il est un humain, et aussi, puisque lion, un nonhumain, est une phrase qui renvoie à une alternative, à des termes incompatibles et contradictoires. Comme l'orateur se veut cohérent, son discours doit donc vouloir dire autre chose. La figure de style utilisée permet de faire l'économie d'avoir à spécifier ce que tout le monde va bien comprendre par soi-même. C'est donc malgré tout une réponse, même si son caractère problématique saute aux yeux. On ne dit pas «Richard est courageux», mais «Richard est un lion». À l'auditeur d'inférer la première réponse, sur le courage, à partir de ce qui est soulevé comme question dans la seconde. Tous les tropes participent de ce même procédé. Ils soulèvent la question de ce qu'ils veulent dire par l'impossibilité d'être pris au pied de la lettre. Cette impossibilité tient à la forme même de la réponse dans le contexte, même l'ironie. «Hugo est une grande plume» ne peut pas plus être pris littéralement que «"Le" Français aime le vin» ou que "Washington soutient Jérusalem». Cela veut dire autre chose, car «le» Français n'existe pas, il n'y a que «des» Français, et la ville de Washington ne soutient littéralement rien. Ces expressions sont comme des questions adressées à l'auditoire, qu'il doit résoudre par lui-même, en dépit du fait qu'on lui présente une réponse. Celle-ci est, dirons-nous, problématologique: elle traduit une question, que la compréhension a pour charge de résoudre et de traduire autrement, ce qui donne lieu au sens, qui se traduira par une littéralité nouvelle, par une autre réponse. Il faut l'inférer pour la trouver. C'est d'ailleurs ce qui se passe dans tous les mécanismes de compréhension. Celle-ci nous indique ce dont il est question dans les réponses qu'on interprète, ce qui suppose qu'on les interroge pour pouvoir préciser la question sous-jacente. On interroge une réponse pour trouver la question qui s'y trouve enfouie, et cela génère une seconde réponse, qui est parfois un texte. Le dialogue, où les questions implicites de l'auteur sont problématisées à leur tour par l'interlocuteur, qui conteste, appuie ou modifie, représente une véritable dialectique de recherche, que Platon n'eût pas reniée.

Les tropes n'échappent pas non plus à la modalisation en quatre niveaux: de l'identité à l'opposition, la différence s'accroît, et l'on trouve là le parcours qui va de la métaphore à l'ironie en passant par la synecdoque (qui inclut) et la métonymie (qui juxtapose et aligne des propriétés, dont l'une est retenue pour toutes les autres, qui résument donc le Sujet, dont elle sert de référent). Dans tous ces tropes, des clauses relatives se voient télescopées. Explicitées, elles seraient introduites par des pronoms interrogatifs, qui servent à dénommer une propriété 
certes, mais surtout une question qui, dans le trope, est présentée comme abolie et évacuée, par économie ou facilité. "Hugo est une grande plume», par exemple, repose sur une interrogative qui dit que les écrivains sont des êtres qui se servent d'une plume. On télescope le qui. «Washington», qui est la capitale des États-Unis, «soutient Jérusalem» est un raccourci pour dire que l'État américain aide Israël. Ce sont des exemples parmi d'autres qui illustrent bien ce mécanisme d'effacement des interrogatifs. Chaque fois, le trope fait l'économie de la formule générale «P (sujet), qui est $\mathrm{Q}$ (propriété),» ou «Q, qui est $\mathrm{P}$,» affirmant simplement que $\mathrm{P}$ est $\mathrm{Q}$. On remplace l'un par l'autre, donc $\mathrm{P}$ par $\mathrm{Q}$, ou $\mathrm{Q}$ par $\mathrm{P}$. «Boire un verre" pour dire «Boire la boisson qui est dans le verre» est un exemple de plus, qui résume l'évacuation de la clause interrogative, de la question ainsi présentée comme ne faisant plus problème. Les qui, les quoi, les où, les quand sont des catégories, au sens où l'entendait Aristote, et plutôt qu'à spécifier toutes ces déterminations d'une interrogation possible, et parfois très longue, sur ce dont on parle en dernière analyse (qui est impossible, elle est infinie), le trope les résume par une seule qualification, qui est métaphorique, métonymique ou synecdochique. «Boire un verre $=$ boire du vin" est une métonymie, dont la fonction est de présenter comme résolu et évident le problème qui n'est pas mentionné, par exemple parce qu'il n'est pas toujours de bon ton de boire (de l'alcool). On évacue donc la question par une réponse qui le permet: la métonymie. Dire que Washington soutient Jérusalem a exactement la même fonction. Qui soutient qui? C'est une question dont la réponse est: les politiques. Donc, on évacue ce problème par la mention des capitales politiques des États concernés. Un trope crée de l'identité, mais il traduit une problématicité plus grande que dans les figures de mots ou même de construction.

Sur la flèche de la problématicité croissante, on trouve au bout du parcours ce qu'on appelle les figures de pensée. Le problème ne peut plus être évacué, pas même grâce à une littéralité impossible, rassemblée dans le trope, qui avale la question, tout en la reflétant. Le problème y est exprimé, même si c'est pour affirmer et soutenir qu'on a la solution.
On avait parlé de quatre opérations. On les trouve dans les tropes, car de l'identité à l'opposition, on a l'ajout et la requalification. Les figures de mots et les figures de construction présentent le même échelonnement. Les figures de pensée, elles, prennent ces opérations pour leur propre objet, elles en traitent; cette fois c'est même la matière du discours: on ironise, on se rétracte, on requalifie, on recule, on avance, on nuance, et on dit qu'on le fait ( ne me fais pas dire...»). On joue aussi sur l'ad hominem: «vous qui êtes un expert, vous ne me direz pas que...», ou «moi qui n'y connais rien, je sais pourtant que...", etc., où c'est la distance entre les individus qui est invoquée pour présenter une solution qui, ainsi, doit s'imposer comme allant de soi. Les figures de pensée portent sur la problématicité d'une question pour ceux qui se la posent. Cela signifie que l'orateur peut opérer aussi bien sur la question que sur ce qu'elle représente pour le locuteur, en atténuant la problématique de cette question, qui oppose et sépare les protagonistes. La technique qui consiste à véhiculer une réponse sans la dire comme dans les "questions rhétoriques», à l'atténuer en faisant comme si l'interlocuteur devait arriver à la même conclusion (c'est la question rhétorique, chère à la démarche oratoire de Nicolas Sarkozy "Vous voulez vivre dans l'insécurité? Alors..." effectivement, qui va répondre par l'affirmative?) sont autant de figures de pensée redoutable. La concession, qui semble donner raison à l'autre, la prétérition, qui évoque ce dont on ne parlera pas, comme pour mieux rappeler que c'est malgré tout important, sans avoir à appuyer sur le clou, parce que cela peut paraître importun ou de mauvais goût, sont autant utilisées que l'opposition qu'on résout, ou que la mise en évidence qu'on rappelle. Le problématique d'une question est littéralement pris en charge, altéré, nuancé, modulé, contré, mais chaque fois, traité comme résolu dans la figure de pensée, alors même qu'elle l'énonce directement. Le caractère problématique est forcément plus élevé que dans le trope, où l'on délittéralise la question. Ici, il y a débat, et il faut l'affronter. Mais non sans technique. Et l'ad hominem (la menace est un bon argument, mais pour les logiciens de l'argumentation, elle est sans validité, car c'est une fallacy, un constat d'impureté 
logique qui ne change rien à sa force argumentative) est souvent invoqué pour s'atteler à la distance entre les individus, si s'attaquer à la question seule ne suffit pas, par la modification de la réponse, l'ajout d'une autre, la répétition ou l'opposition. L'orateur déplace le problème sur les individus, et l'on retrouve ces mêmes opérations de requalification, d'amplification des qualités (ou de diminution), d'opposition ou de confirmation de ce qu'on est ou de ce que l'autre est. Ainsi, sur le plan ad rem, la litote ou l'euphémisme atténue le propos ("Je ne souhaite pas vous voir» pour «Sortez de ma vue!»), et elle correspond au chleuasme sur le plan ad hominem, qui joue sur la distance intersubjective. "Je m'avoue quelque peu maladroit» est ainsi une réponse qui exprime bien la minimisation de l'importance de l'orateur, pour ne pas trop indisposer celui à qui l'on va quand même présenter la solution qu'on a choisie. C'est ce qui fait qu'on peut toujours traduire une figure de pensée ad rem en ad hominem, et inversement. Prenons le cas de la prétérition: «Dois-je vous parler de ceci, que vous connaissez déjà?». Bien sûr que non, si l'interlocuteur le connaît déjà. On hésite, on n'affirme pas haut et fort, on ne met pas trop en difficulté l'interlocuteur, mais l'on ne sait jamais qu'il aurait quand même oublié ce qui est important, ou qu'il ignorerait certaines réponses.

\section{L'ARTICULATION DES FIGURES ET DES OPÉRATIONS}

C'est peut-être le moment le plus captivant de notre argumentation. On l'a dit: de l'identité à la différence, les réponses, en rhétorique, se modulent en quatre opérations de base, mais la modulation est en fait continue, d'où les longs catalogues de figures qui se veulent comme des points d'arrêts. Cela donne le tableau présenté au bas de la page.

La ligne en pointillé, la bissectrice, est un axe hypothétique qui corrèle exactement la problématicité croissante en une distance croissante, proportionnelle, entre les individus concernés. Il n'y a aucune raison que les deux se recouvrent. C'est donc un pur cas de figure, car une telle linéarité n'existe pas par ellemême. On peut exprimer de l'opposition par des figures de son, comme des cris d'hostilité, de même qu'il n'y a aucune raison de penser que toutes les figures de pensée traduisent une conflictualité plus forte, par nature, que des métaphores insultantes, parce que la densité problématique serait élevée.

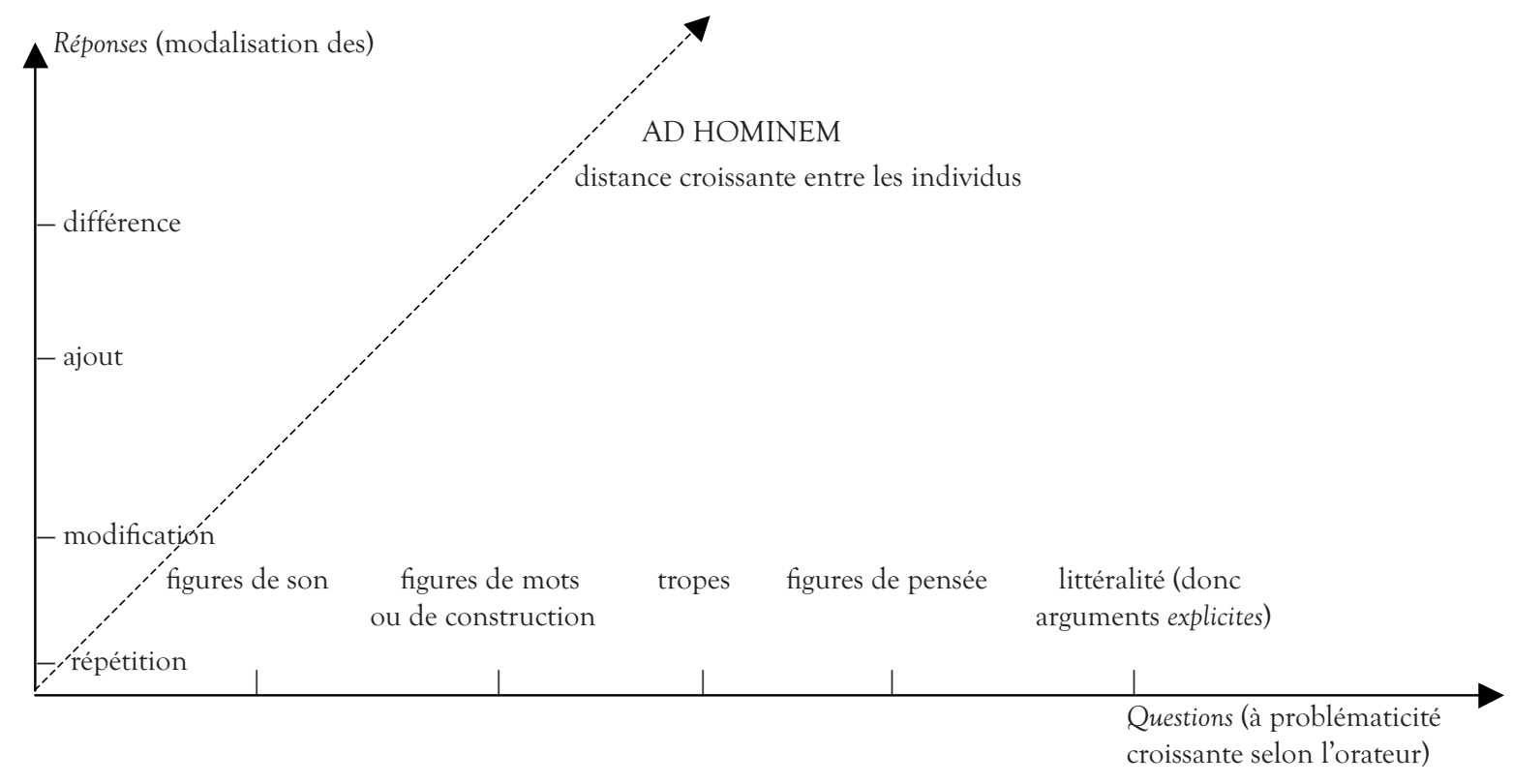


Envisageons quelques cas.

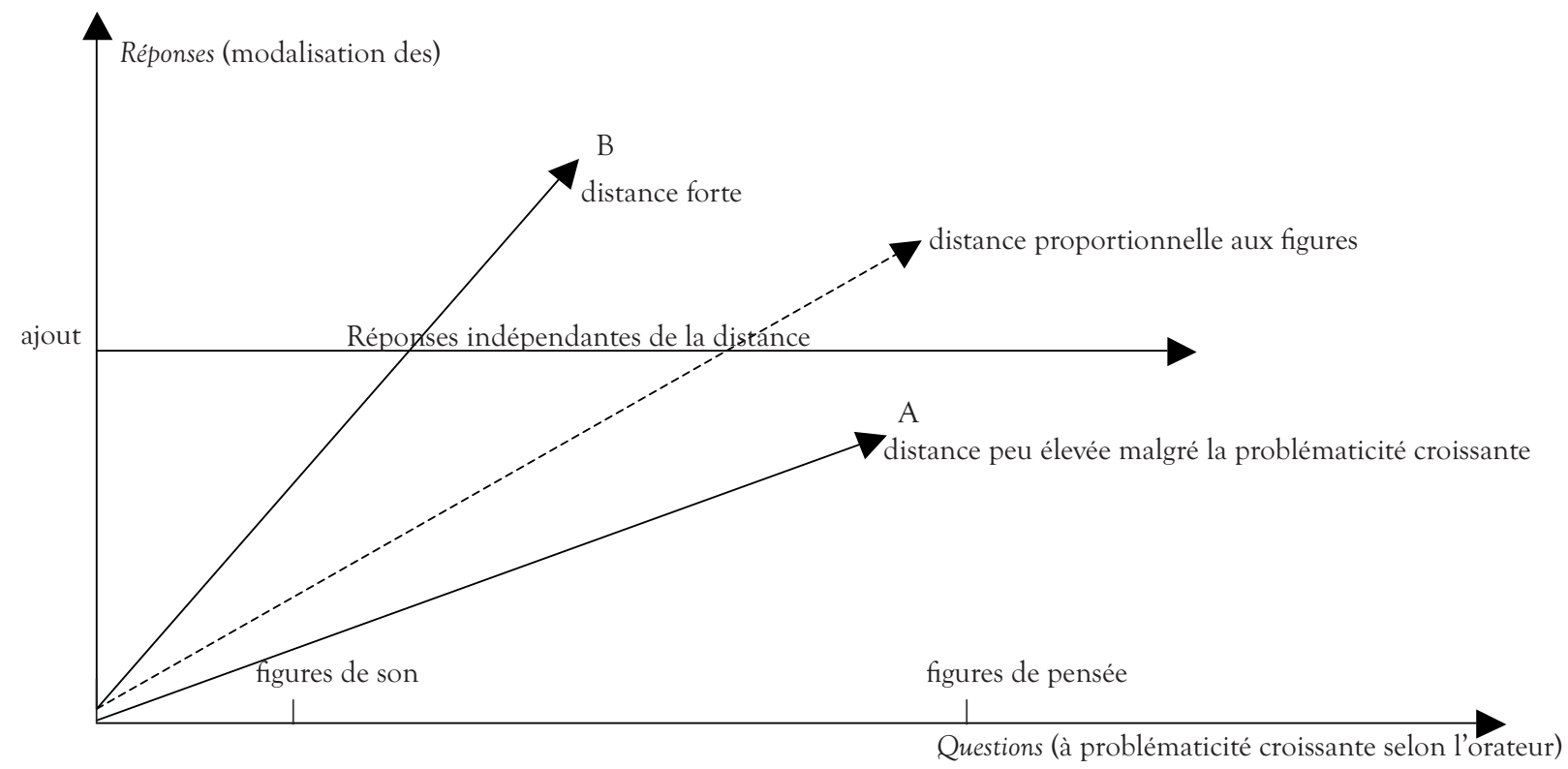

La rhétorique présente une indépendance certaine à chaque niveau par rapport à la distance entre les individus. Ce que traduit la ligne horizontale, pour l'ajout par exemple. Avec les formes qui sont des ajouts, dans le jeu de mots, dans les tropes, ou dans les figures de pensée, la rhétorique est clairement autonome par rapport à la distance intersubjective. Formellement parlant, que ce soit par des figures de son ou de pensée, seule la problématicité semble compter. D'autre part, celle-ci n'est pas forcément signe d'une distance plus grande entre les individus, car à problématicité constante, des jeux de mots ou sur les mots peuvent être plus agressifs, comme dans les cris d'hostilité. C'est seulement pour A qu'on a des figures qui créent peu de distance, tandis que sur la ligne $\mathrm{B}$, l'usage des figures instaure un plus grand écart entre les protagonistes. Il ne faut jamais oublier que certaines figures dites d'opposition peuvent créer une grande communion par rapport à ce à quoi l'on s'oppose.

Pensons à la réplique de Le Pen «Durafourcrématoire», qu'il a proférée quand Durafour avait clamé haut et fort qu'il fallait détruire Le Pen, pour qu'il disparaisse enfin et une fois pour toutes de la vie politique française. On pourrait croire qu'il s'agît là d'une réponse à haute densité problématique. Il n'en est rien, pour la simple raison que ce jeu de mots de mauvais goût est, pour son auteur, l'expression d'une évidence et ne souffre d'aucune problématicité: le moralisateur Durafour veut l'exterminer, comme on exterminait les Juifs dans les fours durant la Deuxième Guerre mondiale. C'est lui qui est présenté comme un nazi, et non Le Pen. La problématicité est du fait de l'auditoire, et cela n'empêche pas le propos de Le Pen de traduire, davantage que ne le fait généralement une figure de son, une modification par l'ironie de ce que disait Durafour. On retrouve ainsi l'axe B du graphique ci-dessus.

\section{CONCLUSION}

La rhétorique modalise la façon dont elle couvre et recouvre ce dont il est question, avalant celle-ci par des réponses qui peuvent être plus ou moins problématiques, le tout pouvant mettre plus ou moins en question l'interlocuteur, selon les valeurs et les points de vue de celui-ci, qui sont extérieurs au langage et à l'énonciation, mais que le locuteur ignore rarement, ne fût-ce que dans ses stratégies de persuasion ou de séduction. 